\title{
The Sum Degree Distance and the Product Degree Distance of Generalized Transformation Graphs $\mathbf{G}^{\text {ab }}$
}

\author{
Keerthi G.Mirajkar ${ }^{*}$ and Priyanka Y. B ${ }^{1}$ \\ Department of Mathematics, Karnatak Arts College, Dharwad-580 001, Karnataka, India \\ *keerthi.mirajkar@gmail.com, 1priyankaybpriya@gmail.com
}

Keywords: Degree distance, Transformation graph $G^{a b}$, Line splitting graph.

\begin{abstract}
In this contribution, we consider line splitting graph $L_{s}(G)$ of a graph $G$ as transformation graph $G^{++}$of $G^{a b}$. We investigate the sum degree distance $D D_{+}(G)$ and product degree distance $D D_{*}(G)$ of transformation graph $G^{a b}$, which are weighted version of Wiener index. The Transformation graphs of $G^{a b}$ are $G^{++}, G^{+-}, G^{-+}$and $G^{--}$.
\end{abstract}

\section{Introduction}

Throughout this paper, we consider finite, un-directed, simple, connected, r-regular graphs with vertex set $V(G)=\left\{v_{1}, v_{2}, v_{3}, \ldots, v_{n}\right\}$ and edge set $E(G)=\left\{e_{1}, e_{2}, e_{3}, \ldots, e_{m}\right\}$. For the undefined terminologies we refer[8].

The degree of vertex in a graph $\mathrm{G}$ is denoted by $\operatorname{deg}_{\mathrm{G}}(\mathrm{v})$ or $\mathrm{d}_{\mathrm{G}}(\mathrm{v})$ and the distance between two vertices $v_{i}$ and $v_{j}$, denoted by $\operatorname{dist}_{G}\left(v_{i}, v_{j}\right)$ or $d_{G}\left(v_{i}, v_{j}\right)$, is the length of a shortest path between the vertices $v_{i}$ and $v_{j}$ in G. The shortest $v_{i}-v_{j}$ path is often called a geodesic. The diameter of a connected graph $\mathrm{G}$ is the length of any longest geodesic. The graphs considered in this construction are with diam $\leq 2$. The degree of an edge $e_{i}$ in $\mathrm{G}$ is the number of edges adjacent to $e_{i}$ and is denoted by $\operatorname{deg}_{G}\left(e_{i}\right)$. The degree of edge in a graph $\mathrm{G}$ is

$$
\operatorname{deg}_{G}\left(e_{i}\right)=\operatorname{deg}_{G}(u v)=\operatorname{deg}_{G}(u)+\operatorname{deg}_{G}(v)-2 .
$$

Topological indices and graph invariants based on distances between vertices of a graph are widely used in mathematical chemistry[2], which are due to their correlations with physical, chemical and thermodynamic parameters of chemical compounds.

One of the oldest and well studied distance based graph invariant associated with a connected graph $G$ is the Wiener number $W(G)$, also termed as Wiener index in chemical or mathematical chemistry literature, which is defined in [13] as the sum of distances over all unordered vertex pairs in $\mathrm{G}$,

i.e.,

$$
W(G)=\sum_{i \leq j} d\left(v_{i}, v_{j}\right)
$$

Which was first time introduced by Wiener. Initially, the Wiener index $W(G)$ was considered as a molecular structure descriptor used in chemical applications, but soon it attracted the interest of pure mathematicians[1,3,5,14,15].

Eventually a number of modifications of the Wiener index were proposed, which are as follows.

$$
\begin{aligned}
& D D_{+}(G)=\sum_{u, v \subseteq V(G)}\left[\operatorname{deg}_{G}(u)+\operatorname{deg}_{G}(v)\right] d_{G}(u, v) \\
& D D_{*}(G)=\sum_{u, v \subseteq V(G)}\left[\operatorname{deg}_{G}(u) \cdot \operatorname{deg}_{G}(v)\right] d_{G}(u, v)
\end{aligned}
$$


The graph invariants defined in (2) and (3) have all been much studied in the past. The invariant $D D_{+}$was first time introduced by Dobrynin and Kochetova[4] and named as sum-degree distance. Later the same quantity was examined under the name "Schulz index" [7]. For mathematical research on degree distance see[9,12] and the references cited therein. A remarkable property of $D D_{+}$is that in the case of trees of order n, the identity $D D_{+}=4 \mathrm{~W}-\mathrm{n}(\mathrm{n}-1)$ holds [10].

Gutman [7] proved that the multiplicative variant of the degree distance, namely $D D_{*}$, i.e., from (2), obeys an analogous relation: $D D_{*}=4 \mathrm{~W}-(2 \mathrm{n}-1)(\mathrm{n}-1)$. This latter quantity is sometimes referred to as the "Gutman index"[6], but here we call it product-degree distance. The open neighborhood $N\left(e_{i}\right)$ of an edge $e_{i}$ in $E(G)$ is the set of edges adjacent to $e_{i}$

$$
\text { i.e, } \quad N\left(e_{i}\right)=\left\{e_{i} / e_{i}, e_{j} \text { are adjacent in } \mathrm{G}\right\} \text {. }
$$

For each edge $e_{i}$ of $\mathrm{G}$, a new vertex $e_{i}^{\prime}$ is taken and the resulting set of vertices is denoted by $E^{\prime}(G)$.

The line Splitting graph $L_{s}(G)$ of a graph $G$ is defined as the graph having vertex set $E(G) \cup E^{\prime}(G)$ with two vertices adjacent if they correspond to adjacent edges of $\mathrm{G}$ or one corresponds to an element $e_{i}^{\prime}$ of $E^{\prime}(G)$ and the other to an element $e_{j}$ of $\mathrm{E}(\mathrm{G})$, and $e_{j}$ is in $N\left(e_{i}\right)$. This concept was introduced by Kulli and Biradar in[11].

\section{Generalized Transformation Graphs $G^{a b}$}

Let $\mathrm{G}=(\mathrm{V}, \mathrm{E})$ be a graph. Let $\alpha, \beta$ and $\alpha^{\prime}, \beta^{\prime}$ be the element of $E(G)$ and $E^{\prime}(G)$ respectively. We say that the associativity of $\alpha$ and $\beta$ is + , if they are adjacent in $\mathrm{G}$ otherwise is and the associativity of $\alpha$ and $\beta^{\prime}$ or $\alpha^{\prime}$ and $\beta$ is + , if $\alpha$ is the neighborhood point of $\beta$ or $\beta$ is neighborhood point of $\alpha$ in $\mathrm{G}$, otherwise is - .

Let $\mathrm{ab}$ be a 2-permutation of the set $\{+,-\}$. We say that $\alpha$ and $\beta$ corresponds to the first term a of ab, and $\alpha, \beta \in \mathrm{E}(\mathrm{G})$. Whereas $\alpha$ and $\beta^{\prime}$ or $\beta$ and $\alpha^{\prime}$ corresponds to the both first and second term of ab and $\alpha^{\prime}, \beta^{\prime} \in E^{\prime}(G)$.

The transformation graph $G^{a b}$ of a graph $\mathrm{G}$ is the graph with vertex set $E(G) \cup E^{\prime}(G)$. $\alpha$ and $\beta$ or $\alpha$ and $\beta^{\prime}$ or $\beta$ and $\alpha^{\prime}$ are adjacent if and only if the following conditions holds;

* $\alpha, \beta \in E(G), \alpha$ and $\beta$ are adjacent in $G$ if $\mathrm{a}=+$ otherwise $\mathrm{a}=-$.

** $\alpha, \beta \in E(G)$ and $\alpha^{\prime}, \beta^{\prime} \in E^{\prime}(G)$, if $\alpha$ neighborhood points of $\beta$ or $\beta$ is neighborhood point of $\alpha$ in $\mathrm{G}$ then $\mathrm{b}=+$ otherwise $\mathrm{b}=-$.

Since there are four distinct 2-permutations of $\{+,-\}$, we obtain 4-graphical transformations of G. Here we consider $G^{++}$, which is nothing but line splitting graph of $\mathrm{G}$ and the other generalized transformation graphs are $G^{+-}, G^{-+}$and $G^{--}$.

Note that, in this paper we consider graphs with $\mathrm{n} \geq 5$ for $G^{++}$and $G^{-+}$and in particular for $G^{+-}$and $G^{--}$we consider graphs with $\mathrm{n}>5$ and having atleast three edges $\mathrm{e}_{\mathrm{i}}, \mathrm{e}_{\mathrm{j}}$ and $\mathrm{e}_{\mathrm{w}} \in \mathrm{E}(\mathrm{G})$; $\mathrm{i}, \mathrm{j}, \mathrm{w}=1,2,3, \ldots, \mathrm{m}$ and $\mathrm{i} \neq \mathrm{j} \neq \mathrm{w}$ such that $\mathrm{e}_{\mathrm{i}}$ and $\mathrm{e}_{\mathrm{j}}$ are non adjacent edges and $\mathrm{e}_{\mathrm{w}}$ is non adjacent to $\mathrm{e}_{\mathrm{i}}$ and $\mathrm{e}_{\mathrm{j}}$.

The aim of present work is to obtain the expression for the sum degree distance and product degree distance of the generalized transformation graphs $G^{a b}$.

\section{Results}

In this section we obtain the sum degree distance and product degree distance of the transformation graphs $G^{a b}$, which is line splitting graph i.e., $G^{++}$, and its generalized transformation graphs $G^{+-}, G^{-+}, G^{--}$. 
We start by stating the following propositions and observations, needed for proving our main results.

Proposition 3.1 Let $\mathrm{G}$ be an $(\mathrm{n}, \mathrm{m})$ graph. Then by the definition order of $G^{a b}$ is $2 \mathrm{~m}$ and

(i) The size of $G^{++}$is $-m+\frac{1}{2} n r^{2}+2 m(r-1)$.

(ii) The size of $G^{+-}$is $-m^{2}+\frac{1}{2}\left(n r^{2}\right)-2 m r$.

(iii) The size of $G^{-+}$is $\frac{m}{2}[m+2 r-3]$.

(iv) The size of $G^{--}$is $\frac{3}{2} m[m-2 r+1]$.

Proof. Let $\mathrm{G}$ be a $(\mathrm{n}, \mathrm{m})$-graph with regular degree $r$, then

(i) $\mathrm{E}\left(\mathrm{G}^{++}\right)=\mathrm{E}(\mathrm{L}(\mathrm{G}))+\sum_{i=1}^{m} \operatorname{deg}_{G}\left(e_{i}\right)$

$$
\begin{aligned}
& =-\mathrm{m}+\frac{1}{2} \sum_{i=1}^{n} d_{i}^{2}+\sum_{i=1}^{m} \operatorname{deg}_{G}\left(e_{i}\right) \\
& =-\mathrm{m}+\frac{1}{2} n r^{2}+m(2 r-2) \\
\mathrm{E}\left(\mathrm{G}^{++}\right) & =-\mathrm{m}+\frac{1}{2} n r^{2}+2 m(r-1) .
\end{aligned}
$$

(ii) $\mathrm{E}\left(G^{+-}\right)=\mathrm{E}(\mathrm{L}(\mathrm{G}))+\sum_{(\mathrm{uv}) \in \mathrm{E}(\mathrm{G})}$ the edges whichare not inc ident to $\mathrm{u}$ and $\mathrm{v}$ in $\mathrm{G}$

$$
\begin{aligned}
& =-\mathrm{m}+\frac{1}{2} n r^{2}+m(m-2 r+1) \quad[\because G \text { is } r-\text { regular graph }] \\
& =-\mathrm{m}+\frac{1}{2} n r^{2}+m^{2}-2 m r+m \\
\mathrm{E}\left(G^{+-}\right) & =m^{2}+\frac{1}{2} n r^{2}-2 m r .
\end{aligned}
$$

(iii) $\mathrm{E}\left(G^{-+}\right)=\frac{1}{2} \sum_{(u v) \in E(G)}$ the edges whicharenot incident to u and $\mathrm{v}$ in $\mathrm{G}+\sum_{i=1}^{m} \operatorname{deg}_{G}\left(e_{i}\right)$

$$
\begin{array}{r}
=\frac{1}{2} m(m-2 r+1)+m\left(\operatorname{deg}_{G}(u)+\operatorname{deg}_{G}(v)-2\right) \\
{[\because G \text { is r-regulargraph }]}
\end{array}
$$$$
=\frac{1}{2} m^{2}-m r+\frac{1}{2} m+m(2 r-2)
$$

$\mathrm{E}\left(G^{-+}\right)=\frac{m}{2}[m+2 m r-3]$.

(iv) $\mathrm{E}\left(G^{--}\right)=\frac{1}{2} \sum_{(\mathrm{uv}) \in \mathrm{E}(\mathrm{G})}$ the edges whicharenot incident to $\mathrm{u}$ and $\mathrm{v}$ in $\mathrm{G}+$

$$
\sum_{(\mathrm{uv}) \in \mathrm{E}(\mathrm{G})} \text { the edges whichare not incident to } u \text { and } \mathrm{v} \text { in } \mathrm{G}
$$

$$
=\frac{1}{2} m(m-2 r+1)+m(m-2 r+1) \quad[\because G \text { is } r \text {-regular graph }]
$$

$\mathrm{E}\left(G^{--}\right)=\frac{3}{2} m[m-2 r+1]$. 
Proposition 3.2 Let $\mathrm{G}$ be an $(\mathrm{n}, \mathrm{m})$ graph. Then the degree of vertices $\mathrm{e}_{\mathrm{i}}$ and $e_{i}^{\prime}$ of $G^{a b}$ are,

(i) $d_{G^{++}}\left(e_{i}\right)=4(r-1)$ and $d_{G^{++}}\left(e_{i}^{\prime}\right)=2(r-1)$.

(ii) $d_{G^{+-}}\left(e_{i}\right)=(m-1)$ and $d_{G^{+-}}\left(e_{i}^{\prime}\right)=m-2 r+1$.

(iii) $d_{G^{-}}\left(e_{i}\right)=(m-1)$ and $d_{G^{-+}}\left(e_{i}^{\prime}\right)=2(r-1)$.

(iv) $d_{G^{--}}\left(e_{i}\right)=2(m-2 r+1)$ and $d_{G^{-}}\left(e_{i}^{\prime}\right)=(m-2 r+1)$.

Proof. Let $\mathrm{G}$ be a $(\mathrm{n}, \mathrm{m})$-graph with regular degree $\mathrm{r}$, then

(i) $d_{G^{++}}\left(e_{i}\right)=2 \operatorname{deg}_{G}\left(e_{i}\right)=2(2 r-2)=4(r-1)$ and $d_{G^{++}}\left(e_{i}^{\prime}\right)=\operatorname{deg}_{G}\left(e_{i}\right)=2 r-2=2(r-1)$.

(ii) $d_{G^{+-}}\left(e_{i}\right)=\operatorname{deg}_{G}\left(e_{i}\right)+(m-2 r+1)=2 r-2+m-2 r+1=(m-1)$ and $d_{G^{+-}}\left(e_{i}^{\prime}\right)=$ The total number of edges which are not incident to $\mathrm{u}$ and $\mathrm{v}$ in $\mathrm{G}$ and $u v=e_{i}$. $=(m-2 r+1)$.

(iii) $d_{G^{-+}}\left(e_{i}\right)=(m-2 r+1)+\operatorname{deg}_{G}\left(e_{i}\right)=m-2 r+1+2 r-2=(m-1)$ and $d_{G^{-+}}\left(e_{i}^{\prime}\right)=\operatorname{deg}_{G}\left(e_{i}\right)=(2 r-2)=2(r-1)$.

(iv) $d_{G^{-}}\left(e_{i}\right)=2$ (Thetotal numberof edges which are not incident to $\mathrm{u}$ and $\mathrm{v}$ in $\mathrm{G}$ and $u v=e_{i}$ ) $=2(m-2 r+1)$ and

$d_{G^{--}}\left(e_{i}^{\prime}\right)=$ The total number of edges which are not incident to $u$ and $\mathrm{v}$ in $\mathrm{G}$ and $u v=e_{i}$. $=(m-2 r+1)$.

We use Proposition 3.2 for the following observations.

\section{Observation A.}

1. $\mathrm{G}$ be any $(\mathrm{n}, \mathrm{m})$ graph.

If $d_{G^{++}}\left(e_{i}, e_{j}\right)=1$, then

$\sum_{\left(e_{i}, e_{j}\right) \subseteq V\left(G^{++}\right)}\left[\operatorname{deg}_{G^{++}}\left(e_{i}\right)+\operatorname{deg}_{G^{++}}\left(e_{j}\right)\right] d_{G^{++}}\left(e_{i}, e_{j}\right)$ in $G^{++}=8 m(r-1)^{2}$.

If $d_{G^{++}}\left(e_{i}, e_{j}\right)=2$, then

$\sum_{\left(e_{i}, e_{j}\right) \subseteq V\left(G^{++}\right)}\left[\operatorname{deg}_{G^{++}}\left(e_{i}\right)+\operatorname{deg}_{G^{++}}\left(e_{j}\right)\right] d_{G^{++}}\left(e_{i}, e_{j}\right)$ in $G^{++}=16(r-1)\left(m^{2}+m-2 m r\right)$.

2. Let $\mathrm{G}$ be any $(\mathrm{n}, \mathrm{m})$ graph.

If $d_{G^{++}}\left(e_{i}, e_{j}^{\prime}\right)=1$, then

$\sum_{\left(e_{i}, e_{j}^{\prime}\right) \subseteq V\left(G^{++}\right)}\left[\operatorname{deg}_{G^{++}}\left(e_{i}\right)+\operatorname{deg}_{G^{++}}\left(e_{j}^{\prime}\right)\right] d_{G^{++}}\left(e_{i}, e_{j}^{\prime}\right)$ in $G^{++}=12 m(r-1)^{2}$.

If $d_{G^{++}}\left(e_{i}, e_{j}^{\prime}\right)=2$, then

$\sum_{\left(e_{i}, e_{j}^{\prime}\right) \subseteq V\left(G^{++}\right)}\left[\operatorname{deg}_{G^{++}}\left(e_{i}\right)+\operatorname{deg}_{G^{++}}\left(e_{j}^{\prime}\right)\right] d_{G^{++}}\left(e_{i}, e_{j}^{\prime}\right)$ in $G^{++}=12 m(r-1)(m-2 r+2)$.

3. Let $\mathrm{G}$ be any $(\mathrm{n}, \mathrm{m})$ graph.

If $d_{G^{++}}\left(e_{i}^{\prime}, e_{j}^{\prime}\right)=2$, then

$\sum_{\left(e_{i}^{\prime}, e_{j}^{\prime}\right) \subseteq V\left(G^{++}\right)}\left[\operatorname{deg}_{G^{++}}\left(e_{i}^{\prime}\right)+\operatorname{deg}_{G^{++}}\left(e_{j}^{\prime}\right)\right] d\left(e_{i}^{\prime}, e_{j}^{\prime}\right)$ in $G^{++}=8(r-1) \sum_{k=1}^{m}(k-1)$.

and

If $d_{G^{++}}\left(e_{i}^{\prime}, e_{j}^{\prime}\right)=3$ when $\mathrm{r}=2$, then

$\sum_{\left(e_{i}^{\prime}, e_{j}^{\prime}\right) \subseteq V\left(G^{++}\right)}\left[\operatorname{deg}_{G^{++}}\left(e_{i}^{\prime}\right)+\operatorname{deg}_{G^{++}}\left(e_{j}^{\prime}\right)\right] d\left(e_{i}^{\prime}, e_{j}^{\prime}\right)$ in $G^{++}=12 \mathrm{~m}(\mathrm{r}-1)$. 
Theorem 3.3. For any $(n, m)$ graph $G$ with $r \geq 2$, if $r=2$ then

$$
D D_{+}\left(G^{++}\right)=4(r-1)\left[m(7 m+5-11 r)+3 m+n r^{2}+2 \sum_{k=1}^{m}(k-1)\right]
$$

And if $r>2$ then

$$
D D_{+}\left(G^{++}\right)=4(r-1)\left[m(7 m+5-11 r)+n r^{2}+2 \sum_{k=1}^{m}(k-1)\right]
$$

Proof. Let $\mathrm{G}$ be any $(\mathrm{n}, \mathrm{m})$-graph. From Proposition $3.1, \mathrm{G}^{++}$contains $2 \mathrm{~m}$ vertices and $\left(-m+\frac{1}{2} n r^{2}+2 m(r-1)\right)$ edges.

From (2), we have

Therefore,

$$
D D_{+}(G)=\sum_{u, v \subseteq V(G)}\left[\operatorname{deg}_{G}(u)+\operatorname{deg}_{G}(v)\right] d_{G}(u, v)
$$

$$
\begin{aligned}
D D_{+}\left(G^{++}\right)= & \sum_{\left(e_{i}, e_{j}\right) \subseteq V\left(G^{++}\right)}\left[\operatorname{deg}_{G^{++}}\left(e_{i}\right)+\operatorname{deg}_{G^{++}}\left(e_{j}\right)\right] d_{G^{++}}\left(e_{i}, e_{j}\right)+ \\
& \sum_{\left(e_{i}, e_{j}^{\prime}\right) \subseteq V\left(G^{++}\right)}\left[\operatorname{deg}_{G^{++}}\left(e_{i}\right)+\operatorname{deg}_{G^{++}}\left(e_{j}^{\prime}\right)\right] d_{G^{++}}\left(e_{i}, e_{j}^{\prime}\right)+ \\
& \sum_{\left(e_{i}^{\prime}, e_{j}^{\prime}\right) \subseteq V\left(G^{++}\right)}\left[\operatorname{deg}_{G^{++}}\left(e_{i}^{\prime}\right)+\operatorname{deg}_{G^{++}}\left(e_{j}^{\prime}\right)\right] d_{G^{++}}\left(e_{i}^{\prime}, e_{j}^{\prime}\right) .
\end{aligned}
$$

Applying observation $\mathrm{A}$ to the above equation, when $r=2$,

$$
\begin{aligned}
D D_{+}\left(G^{++}\right)= & 8 \mathrm{~m}(r-1)^{2}\left(-m+\frac{1}{2} n r^{2}\right)+16 \mathrm{~m}(\mathrm{r}-1)(m-2 r+1)+12 \mathrm{~m}(r-1)^{2}+12 \mathrm{~m}(\mathrm{r}-1) \\
& (\mathrm{m}-2 \mathrm{r}+2)+12 \mathrm{~m}(\mathrm{r}-1)+8(\mathrm{r}-1) \sum_{k=1}^{m}(k-1) .
\end{aligned}
$$

and $r>2$,

$$
\begin{aligned}
D D_{+}\left(G^{++}\right)= & 8 \mathrm{~m}(r-1)^{2}\left(-m+\frac{1}{2} n r^{2}\right)+16 \mathrm{~m}(\mathrm{r}-1)(m-2 r+1)+12 \mathrm{~m}(r-1)^{2}+12 \mathrm{~m}(\mathrm{r}-1) \\
& (\mathrm{m}-2 \mathrm{r}+2)+8(\mathrm{r}-1) \sum_{k=1}^{m}(k-1) .
\end{aligned}
$$

On simplification, we get $(*)$ and $(* *)$

i.e.,

$$
D D_{+}\left(G^{++}\right)=4(r-1)\left[m(7 m+5-11 r)+3 m+n r^{2}+2 \sum_{k=1}^{m}(k-1)\right]
$$

and

$$
D D_{+}\left(G^{++}\right)=4(\mathrm{r}-1)\left[m(7 m+5-11 r)+n r^{2}+2 \sum_{k=2}^{m}(k-1)\right] .
$$

\section{Observation B.}

1. Let $\mathrm{G}$ be any $(\mathrm{n}, \mathrm{m})$ graph.

If $d_{G^{++}}\left(e_{i}, e_{j}\right)=1$, then

$\sum_{\left(e_{i}, e_{j}\right) \subseteq V\left(G^{++}\right)}\left[\operatorname{deg}_{G^{++}}\left(e_{i}\right) \cdot \operatorname{deg}_{G^{++}}\left(e_{j}^{\prime}\right)\right] d_{G^{++}}\left(e_{i}, e_{j}\right)$ in $G^{++}=16 m(r-1)^{2}\left(-m+\frac{1}{2} n r^{2}\right)$.

If $d_{G^{++}}\left(e_{i}, e_{j}\right)=2$, then

$\sum_{\left(e_{i}, e_{j}\right) \subseteq V\left(G^{++}\right)}\left[\operatorname{deg}_{G^{++}}\left(e_{i}\right) \cdot \operatorname{deg}_{G^{++}}\left(e_{j}\right)\right] d_{G^{++}}\left(e_{i}, e_{j}\right)$ in $G^{++}=32(r-1)^{2}\left(m^{2}+m-2 m r\right)$.

2. Let $\mathrm{G}$ be any $(\mathrm{n}, \mathrm{m})$ graph.

If $d_{G^{++}}\left(e_{i}, e_{j}^{\prime}\right)=1$, then

$\sum_{\left(e_{i}, e_{j}^{\prime}\right) \subseteq V\left(G^{++}\right)}\left[\operatorname{deg}_{G^{++}}\left(e_{i}\right) \cdot \operatorname{deg}_{G^{++}}\left(e_{j}^{\prime}\right)\right] d_{G^{++}}\left(e_{i}, e_{j}^{\prime}\right)$ in $G^{++}=16 m(r-1)^{3} m$. 
If $d_{G^{++}}\left(e_{i}, e_{j}^{\prime}\right)=2$, then

$\sum_{\left(e_{i}, e_{j}^{\prime}\right) \subseteq V\left(G^{++}\right)}\left[\operatorname{deg}_{G^{++}}\left(e_{i}\right) \cdot \operatorname{deg}_{G^{++}}\left(e_{j}^{\prime}\right)\right] d_{G^{++}}\left(e_{i}, e_{j}^{\prime}\right)$ in $G^{++}=16 m(r-1)^{2}(m-2 r+2)$.

3. Let $\mathrm{G}$ be any $(\mathrm{n}, \mathrm{m})$ graph.

If $d_{G^{++}}\left(e_{i}^{\prime}, e_{j}^{\prime}\right)=2$, then

$\sum_{\left(e_{i}^{\prime}, e_{j}^{\prime}\right) \subseteq V\left(G^{++}\right)}\left[\operatorname{deg}_{G^{++}}\left(e_{i}^{\prime}\right) \cdot \operatorname{deg}_{G^{++}}\left(e_{j}^{\prime}\right)\right] d_{G^{++}}\left(e_{i}^{\prime}, e_{j}^{\prime}\right)$ in $G^{++}=8(r-1)^{2} \sum_{k=2}^{m}(k-1)$.

If $d_{G^{++}}\left(e_{i}^{\prime}, e_{j}^{\prime}\right)=3$, when $\mathrm{r}=2$, then

$\sum_{\left(e_{i}^{\prime}, e_{j}^{\prime}\right) \subseteq V\left(G^{++}\right)}\left[\operatorname{deg}_{G^{++}}\left(e_{i}^{\prime}\right) \cdot \operatorname{deg}_{G^{++}}\left(e_{j}^{\prime}\right)\right] d_{G^{++}}\left(e_{i}^{\prime}, e_{j}^{\prime}\right)$ in $G^{++}=12 \mathrm{~m}(\mathrm{r}-1)^{2}$.

Theorem 3.4. For any $(n, m)$ graph $G$ with $r \geq 2$,

when $r=2$

$D D_{*}\left(G^{++}\right)=8(r-1)^{2}\left[2 m(3 m-5 r+2)+n r^{2}+3 m+\sum_{k=2}^{m}(k-1)\right]$

and when $r>2$

$D D_{*}\left(G^{++}\right)=8(r-1)^{2}\left[2 m(3 m-5 r+2)+n r^{2}+3 m+\sum_{k=2}^{m}(k-1)\right]$

Proof. Let $\mathrm{G}$ be any $(\mathrm{n}, \mathrm{m})$-graph. From Proposition 3.1, $\mathrm{G}^{++}$contains $2 \mathrm{~m}$ vertices and $\left(-m+\frac{1}{2} n r^{2}+2 m(r-1)\right)$ edges.

From (3), we have

Therefore,

$$
D D_{*}(G)=\sum_{u, v \subseteq V(G)}\left[\operatorname{deg}_{G}(u) \cdot \operatorname{deg}_{G}(v)\right] d_{G}(u, v)
$$

$$
\begin{aligned}
D D_{*}\left(G^{++}\right)= & \sum_{\left(e_{i}, e_{j}\right) \subseteq V\left(G^{++}\right)}\left[\operatorname{deg}_{G^{++}}\left(e_{i}\right) \cdot \operatorname{deg}_{G^{++}}\left(e_{j}\right)\right] d_{G^{++}}\left(e_{i}, e_{j}\right)+ \\
& \sum_{\left(e_{i}, e_{j}^{\prime}\right) \subseteq V\left(G^{++}\right)}\left[\operatorname{deg}_{G^{++}}\left(e_{i}\right) \cdot \operatorname{deg}_{G^{++}}\left(e_{j}^{\prime}\right)\right] d_{G^{++}}\left(e_{i}, e_{j}^{\prime}\right)+ \\
& \sum_{\left(e_{i}^{\prime}, e_{j}^{\prime}\right)+\subseteq V\left(G^{++}\right)}\left[\operatorname{deg}_{G^{++}}\left(e_{i}^{\prime}\right) \cdot \operatorname{deg}_{G^{++}}\left(e_{j}^{\prime}\right)\right] d_{G^{++}}\left(e_{i}^{\prime}, e_{j}^{\prime}\right) .
\end{aligned}
$$

Applying observation $\mathrm{B}$ to the above equation,

when $r=2$,

$$
\begin{aligned}
D D_{*}\left(G^{++}\right)= & 16(r-1)^{2}\left(-m+\frac{1}{2}\right)+32(r-1)^{2}\left(m^{2}+\mathrm{m}-2 \mathrm{mr}\right)+16 \mathrm{~m}(r-1)^{3}+16 \mathrm{~m}(r-1)^{2} \\
& (\mathrm{~m}-2 \mathrm{r}+2)+12 \mathrm{~m}(\mathrm{r}-1)^{2}+8(r-1)^{2} \sum_{k=2}^{m}(k-1) .
\end{aligned}
$$

When $r>2$

$$
\begin{gathered}
D D_{*}\left(G^{++}\right)=16(r-1)^{2}\left(-m+\frac{1}{2}\right)+32(r-1)^{2}\left(m^{2}+\mathrm{m}-2 \mathrm{mr}\right)+16 \mathrm{~m}(r-1)^{3}+16 \mathrm{~m}(r-1)^{2} \\
(\mathrm{~m}-2 \mathrm{r}+2)+8(r-1)^{2} \sum_{k=2}^{m}(k-1) .
\end{gathered}
$$

On simplification, we get $\left(*^{\prime}\right)$ and $\left(* *^{\prime}\right)$

$$
D D_{*}\left(G^{++}\right)=8(r-1)^{2}\left[2 m(3 m-5 r+2)+n r^{2}+3 m+\sum_{k=2}^{m}(k-1)\right]
$$

and

$$
D D_{*}\left(G^{++}\right)=8(r-1)^{2}\left[2 m(3 m-5 r+2)+n r^{2}+\sum_{k=2}^{m}(k-1)\right]
$$




\section{Observation C.}

1. Let $\mathrm{G}$ be any $(n, m)$ graph.

If $d_{G^{+-}}\left(e_{i}, e_{j}\right)=1$, then

$\sum_{\left(e_{i}, e_{j}\right) \subseteq V\left(G^{+-}\right)}\left[\operatorname{deg}_{G^{+-}}\left(e_{i}\right)+\operatorname{deg}_{G^{+-}}\left(e_{j}\right)\right] d_{G^{+-}}\left(e_{i}, e_{j}\right)$ in $G^{+-}=2(m-1)\left(-m+\frac{1}{2} n r^{2}\right)$.

If $d_{G^{+}}\left(e_{i}, e_{j}\right)=2$, then

$\sum_{\left(e_{i}, e_{j}\right) \subseteq V\left(G^{+-}\right)}\left[\operatorname{deg}_{G^{+-}}\left(e_{i}\right)+\operatorname{deg}_{G^{+-}}\left(e_{j}\right)\right] d_{G^{+-}}\left(e_{i}, e_{j}\right)$ in $G^{+-}=4(m-1)\left(m^{2}+m-2 m r\right)$.

2. Let $G$ be any $(n, m)$ graph.

If $d_{G^{+-}}\left(e_{i}, e_{j}^{\prime}\right)=1$, then

$\sum_{\left(e_{i}, e_{j}^{\prime}\right) \subseteq V\left(G^{+-}\right)}\left[\operatorname{deg}_{G^{+-}}\left(e_{i}\right)+\operatorname{deg}_{G^{+-}}\left(e_{j}^{\prime}\right)\right] d_{G^{+-}}\left(e_{i}, e_{j}^{\prime}\right)$ in $G^{+-}=2 m(m-r)(m-2 r+1)$.

If $d_{G^{+}}\left(e_{i}, e_{j}^{\prime}\right)=2$, then

$\sum_{\left(e_{i}, e_{j}^{\prime}\right) \subseteq V\left(G^{+-}\right)}\left[\operatorname{deg}_{G^{+-}}\left(e_{i}\right)+\operatorname{deg}_{G^{+-}}\left(e_{j}^{\prime}\right)\right] d_{G^{+-}}\left(e_{i}, e_{j}^{\prime}\right)$ in $G^{+-}=8 m(m-r)(r-1)$.

If $d_{G^{+-}}\left(e_{i}, e_{j}^{\prime}\right)=3$, then

$\sum_{\left(e_{i}, e_{j}^{\prime}\right) \subseteq V\left(G^{+-}\right)}\left[\operatorname{deg}_{G^{+-}}\left(e_{i}\right)+\operatorname{deg}_{G^{+-}}\left(e_{j}^{\prime}\right)\right] d_{G^{+-}}\left(e_{i}, e_{j}^{\prime}\right)$ in $G^{+-}=6 m(m-r)$.

3. Let $\mathrm{G}$ be any $(\mathrm{n}, \mathrm{m})$ graph.

If $d_{G}\left(e_{i}^{\prime}, e_{j}^{\prime}\right)=2$, then

$\sum_{\left(e_{i}^{\prime}, e_{j}^{\prime}\right) \subseteq V\left(G^{+-}\right)}\left[\operatorname{deg}_{G^{+-}}\left(e_{i}^{\prime}\right)+\operatorname{deg}_{G^{+-}}\left(e_{j}^{\prime}\right)\right] d_{G^{+-}}\left(e_{i}^{\prime}, e_{j}^{\prime}\right)$ in $G^{+-}=4(m-2 r+1) \sum_{k=2}^{m}(k-1)$.

Theorem 3.5. For any $(\mathrm{n}, \mathrm{m})$ graph $\mathrm{G}$,

$$
\begin{aligned}
D D_{+}\left(G^{+-}\right)= & 2 m[m(3 m-5)-1]+m r[n r-6 m-4 r+8]-n r^{2}+ \\
& 4(m-2 r+1) \sum_{k=1}^{r}(k-1) .
\end{aligned}
$$

Proof. Let $\mathrm{G}$ be any $(\mathrm{n}, \mathrm{m})$-graph. From Proposition $3.1, G^{+-}$contains $2 \mathrm{~m}$ vertices and $\left(-m^{2}+\frac{1}{2}\left(n r^{2}\right)-2 m r\right)$ edges.

From (2), we have

$$
D D_{+}(G)=\sum_{u, v \subseteq V(G)}\left[\operatorname{deg}_{G}(u)+\operatorname{deg}_{G}(v)\right] d_{G}(u, v)
$$

Therefore,

$$
\begin{aligned}
D D_{+}\left(G^{+-}\right)= & \sum_{\left(e_{i}, e_{j}\right) \subseteq V\left(G^{+-}\right)}\left[\operatorname{deg}_{G^{+-}}\left(e_{i}\right)+\operatorname{deg}_{G^{+-}}\left(e_{j}\right)\right] d_{G^{+-}}\left(e_{i}, e_{j}\right)+ \\
& \sum_{\left(e_{i}, e_{j}^{\prime}\right) \subseteq V\left(G^{+-}\right)}\left[\operatorname{deg}_{G^{+-}}\left(e_{i}\right)+\operatorname{deg}_{G^{+-}}\left(e_{j}^{\prime}\right)\right] d_{G^{+-}}\left(e_{i}, e_{j}^{\prime}\right)+ \\
& \sum_{\left(e_{i}^{\prime}, e_{j}^{\prime}\right) \subseteq V\left(G^{+-}\right)}\left[\operatorname{deg}_{G^{+-}}\left(e_{i}^{\prime}\right)+\operatorname{deg}_{G^{+-}}\left(e_{j}^{\prime}\right)\right] d_{G^{+-}}\left(e_{i}^{\prime}, e_{j}^{\prime}\right) .
\end{aligned}
$$

Applying observation $\mathrm{C}$ to the above equation, we get

$$
\begin{aligned}
D D_{+}\left(G^{+-}\right)= & 2 m(m-1)(r-1)+4(m-1)\left(m^{2}+m-2 m r\right)+2(m-r)(m-2 r+1)+ \\
& 8 m(m-r)(r-1)+6 m(m-r)+2 m(m-2 r+1) \sum_{k=1}^{m}(k-2) .
\end{aligned}
$$

On simplification,

$$
\begin{aligned}
D D_{+}\left(G^{+-}\right)= & 2 m[m(3 m-5)-1]+m r[n r-6 m-4 r+8]-n r^{2}+ \\
& 4(m-2 r+1) \sum_{k=1}^{r}(k-1) .
\end{aligned}
$$




\section{Observation D.}

1. Let $\mathrm{G}$ be any $(n, m)$ graph.

If $d_{G^{+-}}\left(e_{i}, e_{j}\right)=1$, then

$\sum_{\left(e_{i}, e_{j}\right) \subseteq V\left(G^{+-}\right)}\left[\operatorname{deg}_{G^{+-}}\left(e_{i}\right) \cdot \operatorname{deg}_{G^{+-}}\left(e_{j}\right)\right] d_{G^{+-}}\left(e_{i}, e_{j}\right)$ in $G^{+-}=(m-1)^{2}\left(-m+\frac{1}{2} n r^{2}\right)$.

If $d_{G^{+}}\left(e_{i}, e_{j}\right)=2$, then

$\sum_{\left(e_{i}, e_{j}\right) \subseteq V\left(G^{+-}\right)}\left[\operatorname{deg}_{G^{+-}}\left(e_{i}\right) \cdot \operatorname{deg}_{G^{+-}}\left(e_{j}\right)\right] d_{G^{+-}}\left(e_{i}, e_{j}\right)$ in $G^{+-}=2(m-1)^{2}\left(m^{2}+m-2 m r\right)$.

2. Let $G$ be any $(n, m)$ graph.

If $d_{G^{+-}}\left(e_{i}, e_{j}^{\prime}\right)=1$, then

$\sum_{\left(e_{i}, e_{j}^{\prime}\right) \subseteq V\left(G^{+-}\right)}\left[\operatorname{deg}_{G^{+-}}\left(e_{i}\right) \cdot \operatorname{deg}_{G^{+-}}\left(e_{j}^{\prime}\right)\right] d_{G^{+-}}\left(e_{i}, e_{j}^{\prime}\right)$ in $G^{+-}=\left(m^{2}-2 m r+2 r-1\right)$

$\left(m^{2}-2 r m+m\right)$.

If $d_{G^{+-}}\left(e_{i}, e_{j}^{\prime}\right)=2$, then

$\sum_{\left(e_{i}, e_{j}^{\prime}\right) \subseteq V\left(G^{+-}\right)}\left[\operatorname{deg}_{G^{+-}}\left(e_{i}\right) \cdot \operatorname{deg}_{G^{+-}}\left(e_{j}^{\prime}\right)\right] d_{G^{+-}}\left(e_{i}, e_{j}^{\prime}\right)$ in $G^{+-}=4\left(m^{2}-2 m r+2 r-1\right)(r-1) m$.

If $d_{G^{+-}}\left(e_{i}, e_{j}^{\prime}\right)=3$, then

$\sum_{\left(e_{i}, e_{j}^{\prime}\right) \subseteq V\left(G^{+-}\right)}\left[\operatorname{deg}_{G^{+-}}\left(e_{i}\right) \cdot \operatorname{deg}_{G^{+-}}\left(e_{j}^{\prime}\right)\right] d_{G^{+-}}\left(e_{i}, e_{j}^{\prime}\right)$ in $G^{+-}=3 m\left(m^{2}-2 m+2 r-1\right)$.

3. Let $\mathrm{G}$ be any $(\mathrm{n}, \mathrm{m})$ graph.

If $d_{G^{+-}}\left(e_{i}^{\prime}, e_{j}^{\prime}\right)=2$, then

$\sum_{\left(e_{i}^{\prime}, e_{j}^{\prime}\right) \subseteq V\left(G^{+-}\right)}\left[\operatorname{deg}_{G^{+-}}\left(e_{i}^{\prime}\right) \cdot \operatorname{deg}_{G^{+-}}\left(e_{j}^{\prime}\right)\right] d_{G^{+-}}\left(e_{i}^{\prime}, e_{j}^{\prime}\right)$ in $G^{+-}=2(m-2 r+2)^{2} \sum_{k=2}^{m}(k-1)$.

Theorem 3.6. For any $(n, m)$ graph $G$,

$$
\begin{aligned}
D D_{*}\left(G^{+-}\right)= & m\left[m^{2}(3 m-3)-7(m+1)\right]+\frac{1}{2} n r^{2}\left(m^{2}-m+1\right)-2 m r[2 m(3-r)+2 r-3]+ \\
& 2(m-2 r+2)^{2} \sum_{k=2}^{m}(k-1)^{2} .
\end{aligned}
$$

Proof. Let $\mathrm{G}$ be any $(\mathrm{n}, \mathrm{m})$-graph. From Proposition 3.1, $G^{+-}$contains $2 \mathrm{~m}$ vertices and $-m^{2}+\frac{1}{2}\left(n r^{2}\right)-2 m r$ edges

From (3), we have

Therefore,

$$
D D_{*}(G)=\sum_{u, v \subseteq V(G)}\left[\operatorname{deg}_{G}(u) \cdot \operatorname{deg}_{G}(v)\right] d_{G}(u, v)
$$

$$
\begin{aligned}
D D_{*}\left(G^{+-}\right)= & \sum_{\left(e_{i}, e_{j}\right) \subseteq V\left(G^{+-}\right)}\left[\operatorname{deg}_{G^{+-}}\left(e_{i}\right) \cdot \operatorname{deg}_{G^{+-}}\left(e_{j}\right)\right] d_{G^{+-}}\left(e_{i}, e_{j}\right)+ \\
& \sum_{\left(e_{i}, e_{j}^{\prime}\right) \subseteq V\left(G^{+-}\right)}\left[\operatorname{deg}_{G^{+-}}\left(e_{i}\right) \cdot \operatorname{deg}_{G^{+-}}\left(e_{j}^{\prime}\right)\right] d_{G^{+-}}\left(e_{i}, e_{j}^{\prime}\right)+ \\
& \sum_{\left(e_{i}^{\prime}, e_{j}^{\prime}\right) \subseteq V\left(G^{+-}\right)}\left[\operatorname{deg}_{G^{+-}}\left(e_{i}^{\prime}\right) \cdot \operatorname{deg}_{G^{+-}}\left(e_{j}^{\prime}\right)\right] d_{G^{+-}}\left(e_{i}^{\prime}, e_{j}^{\prime}\right) .
\end{aligned}
$$

Applying observation $D$ to the above equation, we get

$$
\begin{aligned}
D D_{*}\left(G^{+-}\right)= & (m-1)^{2}\left(-m+\frac{1}{2} n r^{2}\right)+2(m-1)^{2}\left(m^{2}+m-2 m r\right)+ \\
& \left(m^{2}-2 m r+2 r-1\right)(m-2 r+1)+4\left(m^{2}-2 m r+2 r-1\right)(r-1) m+ \\
& 3 m\left(m^{2}-2 m+2 r-1\right)+2(m-2 r+1)^{2} \sum_{k=1}^{r}(k-1) .
\end{aligned}
$$


On simplification,

$$
\begin{aligned}
D D_{*}\left(G^{+-}\right)= & m\left[m^{2}(3 m-3)-7(m+1)\right]+\frac{1}{2} n r^{2}\left(m^{2}-m+1\right)-2 m r[2 m(3-r)+2 r-3]+ \\
& 2(m-2 r+2)^{2} \sum_{k=2}^{m}(k-1)^{2} .
\end{aligned}
$$

\section{Observation E.}

1. Let $\mathrm{G}$ be any $(n, m)$ graph.

If $d_{G^{-+}}\left(e_{i}, e_{j}\right)=1$, then

$\sum_{\left(e_{i}, e_{j}\right) \subseteq V\left(G^{-+}\right)}\left[\operatorname{deg}_{G^{-+}}\left(e_{i}\right)+\operatorname{deg}_{G^{-+}}\left(e_{j}\right)\right] d_{G^{-+}}\left(e_{i}, e_{j}\right)$ in $G^{-+}=(m-2 r+1)(m-1) m$.

If $d_{G^{-+}}\left(e_{i}, e_{j}\right)=2$, then

$\sum_{\left(e_{i}, e_{j}\right) \subseteq V\left(G^{-+}\right)}\left[\operatorname{deg}_{G^{-+}}\left(e_{i}\right)+\operatorname{deg}_{G^{-+}}\left(e_{j}\right)\right] d_{G^{-+}}\left(e_{i}, e_{j}\right)$ in $G^{-+}=(m-1)(r-1) m$.

2. Let $\mathrm{G}$ be any $(\mathrm{n}, \mathrm{m})$ graph.

If $d_{G^{-+}}\left(e_{i}, e_{j}^{\prime}\right)=1$, then

$\sum_{\left(e_{i}, e_{j}^{\prime}\right) \subseteq V\left(G^{-+}\right)}\left[\operatorname{deg}_{G^{-+}}\left(e_{i}\right)+\operatorname{deg}_{G^{-+}}\left(e_{j}^{\prime}\right)\right] d_{G^{-+}}\left(e_{i}, e_{j}^{\prime}\right)=2 m(m+2 r-3)(r-1)$.

If $d_{G^{-+}}\left(e_{i}, e_{j}^{\prime}\right)=2$, then

$\left.\sum_{\left(e_{i}, e_{j}^{\prime}\right) \subseteq V\left(G^{-+}\right)}\left[\operatorname{deg}_{G^{-+}}\left(e_{i}\right)+\operatorname{deg}_{G^{-+}}\right)\right] d_{G^{-+}}\left(e_{i}, e_{j}^{\prime}\right)=2 m(m+2 r-3)(m-2 r+2)$.

If $d_{G^{-}}\left(e_{i}, e_{j}^{\prime}\right)=3$, then

$\left.\sum_{\left(e_{i}, e_{j}^{\prime}\right) \subseteq V\left(G^{-+}\right)}\left[\operatorname{deg}_{G^{-+}}\left(e_{i}\right)+\operatorname{deg}_{G^{-+}}\right)\right] d_{G^{-+}}\left(e_{i}, e_{j}^{\prime}\right)=3 m(m+2 r-3)$.

3. Let $\mathrm{G}$ be any $(\mathrm{n}, \mathrm{m})$ graph.

If $d_{G^{-+}}\left(e_{i}^{\prime}, e_{j}^{\prime}\right)=2$, then

$\sum_{\left(e_{i}^{\prime}, e_{j}^{\prime}\right) \subseteq V\left(G^{-+}\right)}\left[\operatorname{deg}_{G}\left(e_{i}^{\prime}\right)+\operatorname{deg}_{G}\left(e_{j}^{\prime}\right)\right] d\left(e_{i}^{\prime}, e_{j}^{\prime}\right)$ in $G^{-+}=8(r-1) \sum_{k=2}^{m}(k-1)$.

Theorem 3.7. For any $(n, m)$ graph $G$,

$$
D D_{+}\left(G^{-+}\right)=m[m(3 m-2)-15]-m r[m-4 r-17]+8(r-1) \sum_{k=2}^{m}(k-1) .
$$

Proof. Let $\mathrm{G}$ be any $(\mathrm{n}, \mathrm{m})$-graph, from the Proposition $3.1, G^{-+}$contains $2 \mathrm{~m}$ vertices and $\frac{m}{2}[m+2 r-3]$ edges.

From (2), we have

$$
D D_{+}(G)=\sum_{u, v \subseteq V(G)}\left[\operatorname{deg}_{G}(u)+\operatorname{deg}_{G}(v)\right] d_{G}(u, v)
$$

Therefore,

$$
\begin{aligned}
D D_{+}\left(G^{-+}\right)= & \sum_{\left(e_{i}, e_{j}\right) \subseteq V\left(G^{-+}\right)}\left[\operatorname{deg}_{G^{-+}}\left(e_{i}\right)+\operatorname{deg}_{G^{-+}}\left(e_{j}\right)\right] d_{G^{-+}}\left(e_{i}, e_{j}\right)+ \\
& \sum_{\left(e_{i}, e_{j}^{\prime}\right) \subseteq V\left(G^{-+}\right)}\left[\operatorname{deg}_{G^{-+}}\left(e_{i}\right)+\operatorname{deg}_{G^{-+}}\left(e_{j}^{\prime}\right)\right] d_{G^{-+}}\left(e_{i}, e_{j}^{\prime}\right)+ \\
& \sum_{\left(e_{i}^{\prime}, e_{j}^{\prime}\right) \subseteq V\left(G^{-+}\right)}\left[\operatorname{deg}_{G^{-+}}\left(e_{i}^{\prime}\right)+\operatorname{deg}_{G^{-+}}\left(e_{j}^{\prime}\right)\right] d_{G^{-+}}\left(e_{i}^{\prime}, e_{j}^{\prime}\right) .
\end{aligned}
$$

Applying observation $\mathrm{E}$ to the above equation, we get

$$
\begin{aligned}
D D_{+}\left(G^{-+}\right)= & (m-2 r+1)(m-1) m+(m-1)(r-1) m+2 m(m+2 r-3)(r-1)+ \\
& 2 m(m+2 r-3)(m-2 r+2)+3 m(m+2 r-3)+8(r-1) \sum_{k=2}^{m}(k-1) .
\end{aligned}
$$


On simplification,

$$
D D_{+}\left(G^{-+}\right)=m[m(3 m-2)-15]-m r[m-4 r-17]+8(r-1) \sum_{k=2}^{m}(k-1) .
$$

\section{Observation F.}

1. Let $\mathrm{G}$ be any $(\mathrm{n}, \mathrm{m})$ graph.

If $d_{G^{-+}}\left(e_{i}, e_{j}\right)=1$, then

$\sum_{\left(e_{i}, e_{j}\right) \subseteq V\left(G^{-+}\right)}\left[\operatorname{deg}_{G^{-+}}\left(e_{i}\right) \cdot \operatorname{deg}_{G^{-+}}\left(e_{j}\right)\right] d_{G^{-+}}\left(e_{i}, e_{j}\right)$ in $G^{-+}=\frac{1}{2}(m-1)^{2}\left(m^{2}-2 m r+m\right)$.

If $d_{G^{-+}}\left(e_{i}, e_{j}\right)=2$, then

$\sum_{\left(e_{i}, e_{j}\right) \subseteq V\left(G^{-+}\right)}\left[\operatorname{deg}_{G^{-+}}\left(e_{i}\right) \cdot \operatorname{deg}_{G^{-+}}\left(e_{j}\right)\right] d_{G^{-+}}\left(e_{i}, e_{j}\right)$ in $G^{-+}=2(m-1)^{2}(r-1) m$.

2. Let $\mathrm{G}$ be any $(\mathrm{n}, \mathrm{m})$ graph.

If $d_{G^{-+}}\left(e_{i}, e_{j}^{\prime}\right)=1$, then

$\sum_{\left(e_{i}, e_{j}^{\prime}\right) \subseteq V\left(G^{-+}\right)}\left[\operatorname{deg}_{G^{-+}}\left(e_{i}\right) \cdot \operatorname{deg}_{G^{-+}}\left(e_{j}^{\prime}\right)\right] d_{G^{-+}}\left(e_{i}, e_{j}^{\prime}\right)$ in $G^{-+}=4 m(m r-m-r+1)(r-1)$.

If $d_{G^{-+}}\left(e_{i}, e_{j}^{\prime}\right)=2$, then

$\sum_{\left(e_{i}, e_{j}^{\prime}\right) \subseteq V\left(G^{-+}\right)}\left[\operatorname{deg}_{G^{-+}}\left(e_{i}\right) \cdot \operatorname{deg}_{G^{-+}}\left(e_{j}^{\prime}\right)\right] d_{G^{-+}}\left(e_{i}, e_{j}^{\prime}\right)$ in $G^{-+}=4 m(m r-m-r-1)(m-2 r+2)$.

If $d_{G^{-+}}\left(e_{i}^{\prime}, e_{j}^{\prime}\right)=3$, then

$\sum_{\left(e_{i}, e_{j}^{\prime}\right) \subseteq V\left(G^{-+}\right)}\left[\operatorname{deg}_{G^{-+}}\left(e_{i}\right) \cdot \operatorname{deg}_{G^{-+}}\left(e_{j}^{\prime}\right)\right] d_{G^{-+}}\left(e_{i}, e_{j}^{\prime}\right)$ in $G^{-+}=6 m(m r-m-r+1)$.

3. Let $\mathrm{G}$ be any $(\mathrm{n}, \mathrm{m})$ graph.

If $d_{G^{-}}\left(e_{i}^{\prime}, e_{j}^{\prime}\right)=2$, then

$\sum_{\left(e_{i}^{\prime}, e_{j}^{\prime}\right) \subseteq V\left(G^{-+}\right)}\left[\operatorname{deg}_{G^{-+}}\left(e_{i}^{\prime}\right) \cdot \operatorname{deg}_{G^{-+}}\left(e_{j}^{\prime}\right)\right] d_{G^{-+}}\left(e_{i}^{\prime}, e_{j}^{\prime}\right)$ in $G^{-+}=8(r-1)^{2} \sum_{k=2}^{m}(k-1)$.

Theorem 3.8. For any $(n, m)$ graph $G$,

$$
\begin{aligned}
D D_{*}\left(G^{-+}\right)= & \frac{1}{2} m\left[m^{2}(m-13)-5 m+8\right]+m r[m(5 m-4 r+8)+4 r-13]+ \\
& 8(r-1)^{2} \sum_{k=2}^{m}(k-1) .
\end{aligned}
$$

Proof. Let $\mathrm{G}$ be any (n,m)-graph. From Proposition 3.1, $G^{-+}$contains $2 \mathrm{~m}$ vertices and $\frac{m}{2}[m+2 r-3]$ edges.

From (3), we have

Therefore,

$$
D D_{*}(G)=\sum_{u, v \subseteq V(G)}\left[\operatorname{deg}_{G}(u) \cdot \operatorname{deg}_{G}(v)\right] d_{G}(u, v)
$$

$$
\begin{aligned}
D D_{*}\left(G^{-+}\right)= & \sum_{\left(e_{i}, e_{j}\right) \subseteq V\left(G^{-+}\right)}\left[\operatorname{deg}_{G^{-+}}\left(e_{i}\right) \cdot \operatorname{deg}_{G^{-+}}\left(e_{j}\right)\right] d_{G^{-+}}\left(e_{i}, e_{j}\right)+ \\
& \sum_{\left(e_{i}, e_{j}^{\prime}\right) \subseteq V\left(G^{-+}\right)}\left[\operatorname{deg}_{G^{-+}}\left(e_{i}\right) \cdot \operatorname{deg}_{G^{-+}}\left(e_{j}^{\prime}\right)\right] d_{G^{-+}}\left(e_{i}, e_{j}^{\prime}\right)+ \\
& \sum_{\left(e_{i}^{\prime}, e_{j}^{\prime}\right) \subseteq V\left(G^{-+}\right)}\left[\operatorname{deg}_{G^{-+}}\left(e_{i}^{\prime}\right) \cdot \operatorname{deg}_{G^{-+}}\left(e_{j}^{\prime}\right)\right] d_{G^{-+}}\left(e_{i}^{\prime}, e_{j}^{\prime}\right) .
\end{aligned}
$$

Applying observation $\mathrm{F}$ to the above equation, we get

$$
D D_{*}\left(G^{-+}\right)=\frac{1}{2}(m-1)^{2}\left(m^{2}-2 m r+m\right)+2(m-1)^{2}(r-1) m+4 m(m r-m-r+1)(r-1)
$$




$$
\begin{aligned}
& +4 m(m r-m-r-1)(m-2 r+2)+6 m(m r-m-r+1)+ \\
& 8(r-1)^{2} \sum_{k=2}^{m}(k-1) .
\end{aligned}
$$

On simplification,

$$
\begin{aligned}
D D_{*}\left(G^{-+}\right)= & \frac{1}{2} m\left[m^{2}(m-13)-5 m+8\right]+m r[m(5 m-4 r+8)+4 r-13]+ \\
& 8(r-1)^{2} \sum_{k=2}^{m}(k-1) .
\end{aligned}
$$

\section{Observation $\mathbf{G}$.}

1. Let $\mathrm{G}$ be any $(\mathrm{n}, \mathrm{m})$ graph.

If $d_{G^{--}}\left(e_{i}, e_{j}\right)=1$, then

$\sum_{\left(e_{i}, e_{j}\right) \subseteq V\left(G^{--}\right)}\left[\operatorname{deg}_{G^{--}}\left(e_{i}\right)+\operatorname{deg}_{G^{--}}\left(e_{j}\right)\right] d_{G^{--}}\left(e_{i}, e_{j}\right)$ in $G^{--}=2 m(m-2 r+1)^{2}$.

If $d_{G^{-}}\left(e_{i}, e_{j}\right)=2$, then

$\sum_{\left(e_{i}, e_{j}\right) \subseteq V\left(G^{--}\right)}\left[\operatorname{deg}_{G^{--}}\left(e_{i}\right)+\operatorname{deg}_{G^{--}}\left(e_{j}\right)\right] d_{G^{--}}\left(e_{i}, e_{j}\right)$ in $G^{--}=8(m-2 r+1)(r-1)$.

2. Let $\mathrm{G}$ be any $(\mathrm{n}, \mathrm{m})$ graph.

If $d_{G^{-}}\left(e_{i}, e_{j}^{\prime}\right)=1$, then

$\sum_{\left(e_{i}, e_{j}^{\prime}\right) \subseteq V\left(G^{--}\right)}\left[\operatorname{deg}_{G^{--}}\left(e_{i}\right)+\operatorname{deg}_{G^{--}}\left(e_{j}^{\prime}\right)\right] d_{G^{--}}\left(e_{i}, e_{j}^{\prime}\right)$ in $G^{--}=3 m(m-2 r+1)^{2}$.

if $d_{G^{-}}\left(e_{i}, e_{j}^{\prime}\right)=2$, then

$\sum_{\left(e_{i}, e_{j}^{\prime}\right) \subseteq V\left(G^{--}\right)}\left[\operatorname{deg}_{G^{--}}\left(e_{i}\right)+\operatorname{deg}_{G^{--}}\left(e_{j}^{\prime}\right)\right] d_{G^{--}}\left(e_{i}, e_{j}^{\prime}\right)$ in $G^{--}=6 m(2 r-1)(m-2 r+1)$.

3. Let $\mathrm{G}$ be any $(\mathrm{n}, \mathrm{m})$ graph.

If $d_{G^{-}}\left(e_{i}^{\prime}, e_{j}^{\prime}\right)=2$, then

$$
\sum_{\left(e_{i}^{\prime}, e_{j}^{\prime}\right) \subseteq V\left(G^{--}\right)}\left[\operatorname{deg}_{G^{--}}\left(e_{i}^{\prime}\right)+\operatorname{deg}_{G^{--}}\left(e_{j}^{\prime}\right)\right] d_{G^{--}}\left(e_{i}^{\prime}, e_{j}^{\prime}\right) \text { in } G^{--}=4(m-2 r+1) \sum_{k=2}^{m}(k-1) \text {. }
$$

Theorem 3.9. For any $(n, m)$ graph $G$,

$$
D D_{+}\left(G^{--}\right)=m\left[5 m^{2}-4 m-9\right]-4 m r[5 r-7]+4(m-2 r+1) \sum_{k=2}^{m}(k-1) .
$$

Proof. Let $\mathrm{G}$ be any $(\mathrm{n}, \mathrm{m})$-graph. From proposition $3.1, G^{--}$contains $2 \mathrm{~m}$ vertices and $\frac{3}{2} m[m-2 r+1]$ edges.

From (2), we have

$$
D D_{+}(G)=\sum_{u, v \subseteq V(G)}\left[\operatorname{deg}_{G}(u)+\operatorname{deg}_{G}(v)\right] d_{G}(u, v)
$$

Therefore,

$$
\begin{aligned}
D D_{+}\left(G^{--}\right)= & \left.\sum_{\left(e_{i}, e_{j}\right) \subseteq V\left(G^{--}\right)}\left[\operatorname{deg}_{G^{--}}\left(e_{i}\right)+\operatorname{deg}_{G^{--}}\left(e_{j}\right)\right] d_{G^{--}}\left(e_{i}, e_{j}\right)\right]+ \\
& \left.\sum_{\left(e_{i}, e_{j}^{\prime}\right) \subseteq V\left(G^{--}\right)}\left[\operatorname{deg}_{G^{--}}\left(e_{i}\right)+\operatorname{deg}_{G^{--}}\left(e_{j}^{\prime}\right)\right] d_{G^{--}}\left(e_{i}, e_{j}^{\prime}\right)\right]+ \\
& \left.\sum_{\left(e_{i}^{\prime}, e_{j}^{\prime}\right) V\left(G^{--}\right)}\left[\operatorname{deg}_{G^{--}}\left(e_{i}^{\prime}\right)+\operatorname{deg}_{G^{--}}\left(e_{j}^{\prime}\right)\right] d_{G^{--}}\left(e_{i}^{\prime}, e_{j}^{\prime}\right)\right] .
\end{aligned}
$$

Applying observation $\mathrm{G}$ to the above equation, we get

$$
\begin{aligned}
D D_{+}\left(G^{--}\right)= & 2 m(m-2 r+1)^{2}+8(m-2 r+1)(r-1)+3 m(m-2 r+1)^{2}+6 m(2 r-1)(m-2 r+1) \\
& +4(m-2 r+1) \sum_{k=2}^{m}(k-1)
\end{aligned}
$$


On simplification,

$$
D D_{+}\left(G^{--}\right)=m\left[5 m^{2}-4 m-9\right]-4 m r[5 r-7]+4(m-2 r+1) \sum_{k=2}^{m}(k-1) .
$$

\section{Observation $\mathbf{H}$.}

1. Let $\mathrm{G}$ be any $(\mathrm{n}, \mathrm{m})$ graph.

If $d_{G^{--}}\left(e_{i}, e_{j}\right)=1$, then

$\sum_{\left(e_{i}, e_{j}\right) \subseteq V\left(G^{--}\right)}\left[\operatorname{deg}_{G^{--}}\left(e_{i}\right) \cdot \operatorname{deg}_{G^{--}}\left(e_{j}\right)\right] d_{G^{--}}\left(e_{i}, e_{j}\right)$ in $G^{--}=2 m(m-2 r+1)^{3}$.

If $d_{G^{-}}\left(e_{i}, e_{j}\right)=2$, then

$\sum_{\left(e_{i}, e_{j}\right) \subseteq V\left(G^{--}\right)}\left[\operatorname{deg}_{G^{--}}\left(e_{i}\right) \cdot \operatorname{deg}_{G^{--}}\left(e_{j}\right)\right] d_{G^{--}}\left(e_{i}, e_{j}\right)$ in $G^{--}=8 m(m-2 r+1)^{2}(r-1)$.

2. Let $\mathrm{G}$ be any $(n, m)$ graph.

If $d_{G^{--}}\left(e_{i}, e_{j}^{\prime}\right)=1$, then

$\sum_{\left(e_{i}, e_{j}^{\prime}\right) \subseteq V\left(G^{--}\right)}\left[\operatorname{deg}_{G^{--}}\left(e_{i}\right) \cdot \operatorname{deg}_{G^{--}}\left(e_{j}^{\prime}\right)\right] d_{G^{--}}\left(e_{i}, e_{j}^{\prime}\right)$ in $G^{--}=2 m(m-2 r+1)^{3}$.

If $d_{G^{-}}\left(e_{i}, e_{j}^{\prime}\right)=2$, then

$\sum_{\left(e_{i}^{\prime}, e_{j}^{\prime}\right) \subseteq V\left(G^{--}\right)}\left[\operatorname{deg}_{G^{--}}\left(e_{i}\right) \cdot \operatorname{deg}_{G^{--}}\left(e_{j}^{\prime}\right)\right] d_{G^{--}}\left(e_{i}, e_{j}^{\prime}\right)$ in $G^{--}=4 m(m-2 r+2)^{2}(2 r-1)$.

3. Let $\mathrm{G}$ be any $(\mathrm{n}, \mathrm{m})$ graph.

If $d_{G^{-}}\left(e_{i}^{\prime}, e_{j}^{\prime}\right)=2$, then

$\sum_{\left(e_{i}^{\prime}, e_{j}^{\prime}\right) \subseteq V\left(G^{--}\right)}\left[\operatorname{deg}_{G^{--}}\left(e_{i}^{\prime}\right) \cdot \operatorname{deg}_{G^{--}}\left(e_{j}^{\prime}\right)\right] d_{G^{--}}\left(e_{i}^{\prime}, e_{j}^{\prime}\right)$ in $G^{--}=2(m-2 r+1)^{2} \sum_{k=2}^{m}(k-1)$.

Theorem 3.10. For any $(n, m)$ graph $\mathrm{G}$,

$$
\begin{aligned}
D D_{*}\left(G^{--}\right)= & 2 m\left[m^{2}(m-3)-5 m-6\right]-4 m r\left[3 m^{2}-4 r^{2}+10 m r-14 m+12 r-13\right]+ \\
& 2(m-2 r+1)^{2} \sum_{k=2}^{m}(k-1) .
\end{aligned}
$$

Proof. Let $\mathrm{G}$ be any $(\mathrm{n}, \mathrm{m})$-graph. From Proposition $3.1, G^{--}$contains $2 \mathrm{~m}$ vertices and $\frac{3}{2} m[m-2 r+1]$ edges.

From (3), we have

$$
D D_{*}(G)=\sum_{u, v \subseteq V(G)}\left[\operatorname{deg}_{G}(u) \cdot \operatorname{deg}_{G}(v)\right] d_{G}(u, v)
$$

Therefore,

$$
\begin{aligned}
D D_{*}\left(G^{--}\right)= & \sum_{\left(e_{i}, e_{j}\right) \subseteq V\left(G^{--}\right)}\left[\operatorname{deg}_{G^{--}}\left(e_{i}\right) \cdot \operatorname{deg}_{G^{--}}\left(e_{j}\right)\right] d_{G^{--}}\left(e_{i}, e_{j}\right)+ \\
& \sum_{\left(e_{i}, e_{j}^{\prime}\right) \subseteq V\left(G^{--}\right)}\left[\operatorname{deg}_{G^{--}}\left(e_{i}\right) \cdot \operatorname{deg}_{G^{--}}\left(e_{j}^{\prime}\right)\right] d_{G^{--}}\left(e_{i}, e_{j}^{\prime}\right)+ \\
& \sum_{\left(e_{i}^{\prime}, e_{j}^{\prime}\right) \subseteq V\left(G^{--}\right)}\left[\operatorname{deg}_{G^{--}}\left(e_{i}^{\prime}\right) \cdot \operatorname{deg} g_{G^{--}}\left(e_{j}^{\prime}\right)\right] d_{G^{--}}\left(e_{i}^{\prime}, e_{j}^{\prime}\right) .
\end{aligned}
$$

Applying observation $\mathrm{H}$ the above equation, we get

$$
\begin{aligned}
D D_{*}\left(G^{--}\right)= & 2 m(m-2 r+1)^{3}+8 m(m-2 r+1)^{2}(r-1)+2 m(m-2 r+1)^{3}+ \\
& 4 m(m-2 r+2)^{2}(2 r-1)+2(m-2 r+1)^{2} \sum_{k=2}^{m}(k-1) .
\end{aligned}
$$

On simplification,

$$
\begin{aligned}
D D_{*}\left(G^{--}\right)= & 2 m\left[m^{2}(m-3)-5 m-6\right]-4 m r\left[3 m^{2}-4 r^{2}+10 m r-14 m+12 r-13\right]+ \\
& 2(m-2 r+1)^{2} \sum_{k=2}^{m}(k-1) .
\end{aligned}
$$




\section{Acknowledgement:}

The Author ${ }^{1}$ thankful to University Grants Commission (UGC), Govt. of India for financial support through UGC-RGNF-2014-15-SC-KAR-75098/(SA-III/www.ugc-rgnf.nic.in).

\section{References}

[1] G. Caporossi, M. Paiva, D. Vukičević, M. Segatto, Centrality and betweenness: vertex and edge decomposition of the Wiener index, MATCH Commun. Math. Comput. Chem., 68 (2012) 293-302.

[2] M.V. Diudea, I. Gutman, L. Jäntschi, Molecular Topology, Nova, Huntington, NY, 2001.

[3] A. Dobrynin, R. Entringer, I. Gutman, Wiener index of trees: theory and applications. Acta Appl Math, 66 (2001) 211-249.

[4] A.A. Dobrynin, A.A. Kochetova, Degree distance of a graph: a degree analogue of the Wiener index, J. Chem. Inf. Comput. Sci., 34 (1994) 1082-1086.

[5] R.C. Entringer, D.E. Jackson, D. A. Snyder, Distance in graphs. Czechoslov Math. J., 26 (1976) 283-296.

[6] L. Feng, B.Liu, The maximal Gutman index of bicyclic graphs, MATCH Commun. Math. Comput. Chem., 66 (2011) 699-708.

[7] I. Gutman, Selected properties of the Schultz molecular topological index, J. Chem. Inf. Comput. Sci., 34 (1994) 1087-1089.

[8] F. Harary, Graph Theory, Addison-Wesley, Reading, 1969.

[9] A. Ili c', D. Stevanovic', L. Feng, G. Yu, P. Dankelmann, Degree distance of unicyclic and bicyclic graphs, Discrete Appl. Math., 159 (2011) 779-788.

[10] D.J. Kelein , Z. Mihali c', D. Plavši c' , N.Trinajsti c', Molecular topological index: a relation with the Wiener index, J. Chem. Inf. Comput. Sci., 32 (1992) 304-305.

[11] V. R. Kulli, M. S. Biradar, The line splitting graph of a graph, Acta Ciencia Indica., XXVII, M.No. 3 (2002) 317-322.

[12] I. Tomescu, Ordering connected graphs having small degree distance, Discrete Appl. Math., 158 (2010) 1714-1717.

[13] H. Wiener, Structural determination of paraffin boiling points, J. Am. Chem. Soc., 69 (1947) 17-20.

[14] H. Wiener, Correlation of heats of isomerization and differences in heats of vaporization of isomers among the paraffin hydrocarbons, J. Am. Chem. Soc., 69 (1947) 2636-2638.

[15] H. Wiener, Influence of interatomic forces on paraffin properties, J. Chem. Phys., 15 (1947) $766-766$. 